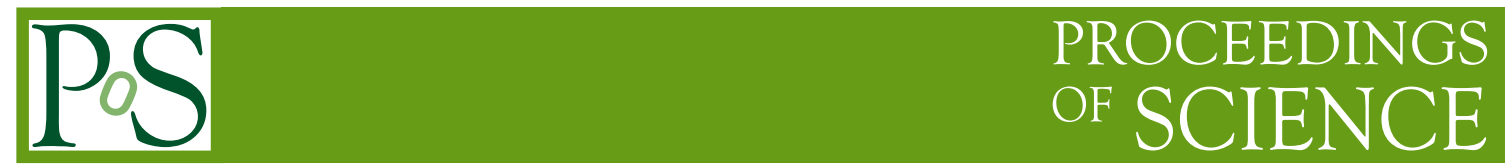

\title{
Late stages of stellar evolution with PLANCK
}

\author{
Grazia Umana*, Corrado Trigilio, Carla S. Buemi \\ INAF- Osservatorio Astrofisico di Catania Italy \\ E-mail: gumanaloact.inaf.it
}

\section{Carlo Burigana}

INAF-IASF Bologna Italy

E-mail: burigana@iasfbo.inaf.it

\section{Paolo Leto}

INAF-IRA Noto(SR) Italy

E-mail: p.letodira.inaf.it

The Planck mission may, in principle, have a large impact on stellar studies because it will cover a very important observational band. For various classes of stellar objects continuum observations at millimetric and submillimetric wavelengths would be very fruitful, because they provide essential clues to understand the physics of these stars and constitute a powerful addition to current studies, which are carried out mostly at centimetric wavelengths. However, the high level of galactic confusion together with the intrinsic low flux density make the detection of such kinds of source quite challenging. In this paper we present first results of a feasibility study aimed to investigate on the possibility to observe with Planck evolved galactic objects belonging to a evolutionary phase that strongly influences the chemical evolution of the Galaxy

$C M B$ and Physics of the Early Universe

20-22 April 2006

Ischia, Italy

\footnotetext{
* Speaker.
} 


\section{From AGB to PNs..going with the wind}

When a low or intermediate mass star is approaching the end of its evolution, it goes through a period of heavy massloss known as Asymptotic Giant Branch (AGB phase).The ejected envelopes are partially condensed in dust grains and completely obscure the central star. Immediately after the AGB phase, the massloss stops and the central object may become optically visible as the dust disperse (Proto Planetary PPNs phase). Eventually, once it reaches a temperature of 20,000-30,000 $\mathrm{K}$, the central star starts to ionize the AGB shell and a Planetary Nebula (PNs phase) will form.

Planck observations are potentially very important for stellar studies, as they cover a scarcely explored observational band. They will be complementary to the radio and to the far-IR data, to fully understand the emission mechanism/s operating in specific classes of stellar objects. In particular, for evolved galactic objects, which trace the transition towards PNs, Planck observations would allow to built the SED of large samples. This, together with an appropriate modelling, will provide important clues for the study the physical properties of their dusty circumstellar envelopes (CSE): mass, temperature, morphology, existence of multiple shells. Such kind of studies are very important in the framework of Galaxy chemical evolution, as PNs and their progenitors are among the major sources of recycled interstellar matter.

\section{Sample selection}

In order to determine the detectability of such sources in the PLANCK channels we first selected our samples from more recent and complete compilations, namely:

- AGB: [5];

- Post-AGB:[3] and from cross correlation between IRAS PSC and SAO [4];

- PNs: Cross correlation between NVSS and galactic catalog [2]

for a total of about 1,700 objects

All the selected candidates have strong signatures in the IR (infrared excess). Preliminary survey of small sample have shown millimetric and sub-millimetric emission up to few Jy. PNs have also a free-free contribution, due to the partially ionization of their CSE, that in same cases can reach a Jy level. Finally, several targets are located at high galactic latitude, where the Galactic noise is reduced.

\section{Are they detectable by Planck?}

To evaluate the chance to detect them with a given $\mathrm{S} / \mathrm{N}$, their intrinsic emission should be compared to the PLANCK nominal sensitivity. The predicted fluxes at PLANCK channels have been evaluated by fitting the IRAS measurements with a BB curve and by extrapolating the dust contribution to the PLANCK channels . For the PNs sample we summed to the dust contribution the free-free radio emission obtained from the NVSS measurements, extrapolated to the Planck channels, assuming an optically thin nebula. In the analysis the nominal instrumental Planck sensitivity per resolution element, $\sigma_{\text {ins }}$, (PLANCK LFI, Instrument Science verification Review, 1999; 


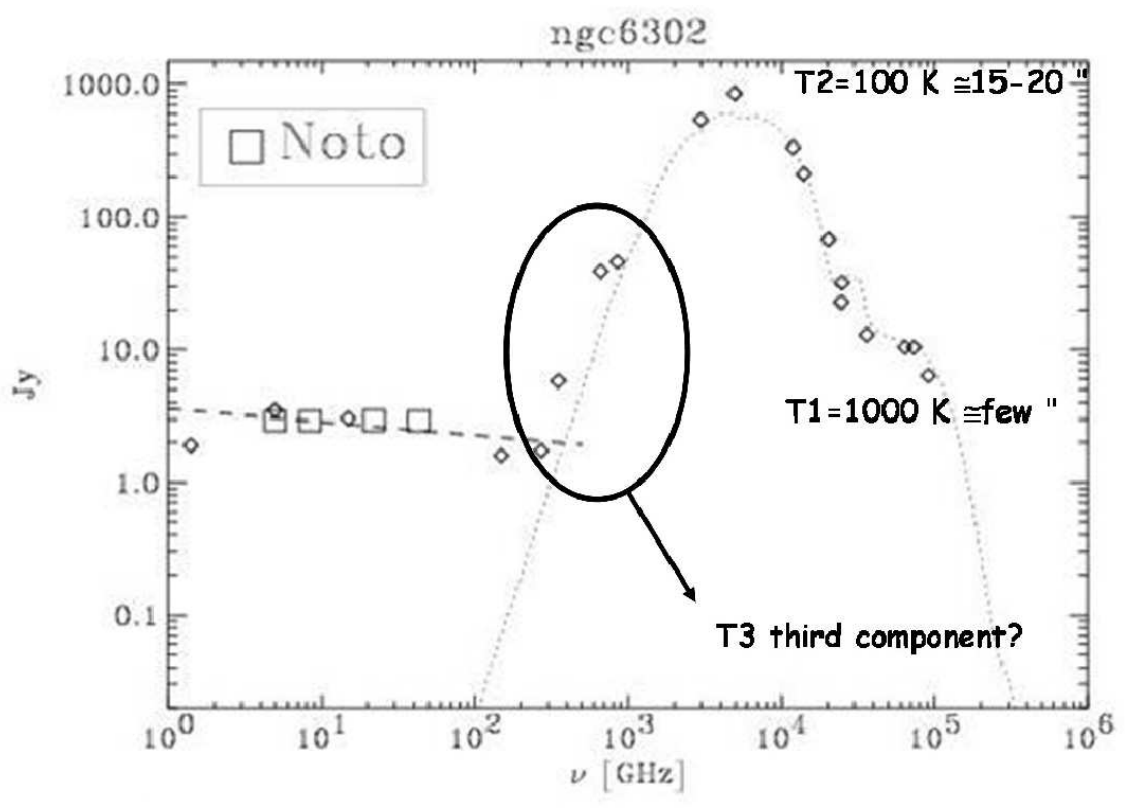

Figure 1: The SED of Planetary Nebula NGC 6302, build with data from the literature and with Noto measurements. The sub-millimetric and infrared part of the SED can be fitted by two (or three) dusty components at different dust temperature

LFI and HFI proposal), the CMB confusion noise, $\sigma_{C M B}$, [1], Galactic, $\sigma_{G A L}$ and extragalactic, $\sigma_{e x-\text { sou }}$ foregrounds confusion noise [6] have been considered as noise sources. Results indicate that a significant number of evolved Galactic object in our samples could be detected during the mission, at least in one of the channels, with a good $\mathrm{S} / \mathrm{N}$ [7].

\section{Ancillary observations}

Focused observations are necessary to gather more information to finally assess our working samples and for a more careful modelling of the expected fluxes in the PLANCK channels. A survey of 69 bright PNs, selected on the basis of their flux at $1.4 \mathrm{GHz}$, has been conducted at 5 , 8.4, 22 and $43 \mathrm{GHz}$ with the Noto32m INAF-IRA radiotelescope. Results have allowed to better define the radio part of the SEDs pointing out:

- A previous underestimation of source count at $22 \mathrm{GHz}$, probably related to the wrong hypothesis of an optically thin nebula at $20 \mathrm{~cm}$.

- The importance of the spectral bands covered by PLANCK as several deviations from the assumed free-free radio spectrum have been observed, indicating that other emission/absorbing effects, besides f-f and thermal emission from dust, may be present.

- The possibility to use radio + PLANCK data to determine the presence of multiple shells in the CSE. 
As examples of our results and of the potentiality of PLANCK data, the SED of NGC 6302 is shown in figure 1. NGC 6302 is one of the very few PNs for which data in the spectral band covered by PLANCK are already available. The presence of multiple dusty shells, with different typical sizes and dust temperatures is evident. Radio and PLANCK data together with an appropriate modelling (DUSTY code) will allow us to determine if multiple shells are present in large sample of postAGB and PNs. The SED of NGC 6369 gives another example of the importance of collecting data in the spectral range covered by PLANCK: an extra process appear to be at work besides f-f and thermal dust as pointed out by the extra-absorption starting from around $30 \mathrm{GHz}$.

Successive steps of our study to better estimate the noise source and the source intrinsic flux will include:

- evaluation of the Galactic confusion noise for different values of Galactic latitude, around source position, from WMAP maps.

- More radio and millimetric observations aimed to better evaluated the SEDs of different objects.

\section{References}

[1] C.L. Bennett, et al., 2003 First-Year Wilkinson Microwave Anisotropy Probe (WMAP) Observations: Preliminary Maps and Basic Results, APJS 1480, 1

[2] J.J. Condon \& D.L. Kaplan Bennett, 1998, Planetary Nebulae in the NRAO VLA Sky Survey, APJS 117,361

[3] P. Garcia-Lario , A. Manchado, W. Pych, and S.R. Pottasch, 1997, Near infrared photometry of IRAS sources with colours like planetary nebulae. III, A\&ASS 126, 479

[4] R. D. Oudmaijer, W.E.C.J. van der Veen, L.B.F.M. Waters, N.R. Trams, C. Waelkens, E. Engelsman, 1992, SAO stars with infrared excess in the IRAS Point Source Catalog, A\&ASS 96, 625

[5] L. A. Nyman et al., 1992, A survey of circumstellar CO emission from a sample of IRAS point sources, A\&ASS 93, 121

[6] L. Toffolatti, F. Argueso Gomez, G. de Zotti, P. Mazzei, L. Danese, C. Burigana, 1998, Extragalactic source counts and contributions to the anisotropies of the cosmic microwave background: predictions for the Planck Surveyor mission, MNRAS 297, 117

[7] G. Umana, C. Burigana and C. Trigilio, 2006 Late stages of stellar evolution with PLANCK: a feasibility study in proceedings of XLIX Congresso della Società Astronomica Italiana G. Leto and F. Zuccarello eds. 